\title{
Marcas olfativas: ¿un derecho evolutivo o restrictivo? Perspectiva del derecho comparado (Honduras-Colombia)
}

José Alexander Ávila Vallecillo ${ }^{1}$

\section{RESUMEN}

El artículo describe, analiza y sintetiza los escritos vinculados al tema de marcas olfativas desde una perspectiva nacional e internacional, tomando como referente jurídico las normas, doctrina y jurisprudencia que rigen a estos signos distintivos, dilucidando a su vez la problemática que enfrentan al solicitar su inscripción ante las oficinas registrales correspondientes. En cuanto a su reconocimiento jurídico, la doctrina internacional se encuentra totalmente dividida, algunos autores consideran que las marcas olfativas deberían ser reconocidas y protegidas como tal, otros se resisten a concebir tal idea. De igual forma, los requerimientos jurídicos que restringen la inscripción de marcas no visibles alrededor del mundo gira en torno a tres requerimientos básicos: distintividad, perceptibilidad y representación gráfica. En ese orden de ideas y con relación a la entrada en vigor del Tratado de Libre Comercio suscrito entre la República Dominicana, Centroamérica y los Estados Unidos (DR-CAFTA), Honduras incorporó a su compendio jurídico la inscripción de signos olfativos a su legislación, por tanto, resulta necesario conocer los requerimientos y razonamientos jurídicos con los cuales la Dirección General de Propiedad Intelectual (DIGEPIH) y la Superintendencia de Industria y Comercio de Colombia se encuentran atendiendo dicha problemática. De hecho, ambas entidades gubernamentales podrían estar amparando sus pronunciamientos denegatorios bajo el umbral de los requisitos básicos inherentes a toda marca y que se evidencian en la sentencia Sieckman, pese al expreso reconocimiento de los olores como signos distintivos a lo interno de sus compendios jurídicos. En síntesis, la investigación pretende establecer un análisis comparado entre Colombia y Honduras con relación a dichas marcas, tomando como referentes jurídicos la ley, la doctrina y jurisprudencia internacional.

Palabras clave: marca olfativa, signos distintivos, dirección general de propiedad intelectual, secretaría de industria y comercio, distintividad, perceptibilidad, y representación gráfica.

1 Profesor universitario, Facultad de Ciencias Jurídicas, Departamento de Derecho Privado, UNAH: jaavhn@yahoo.com 


\section{ABSTRACT}

This paper seeks, analyzes and synthesizes the writings related to the topic of scent marks from a national and international perspective on the legal rules concerning, doctrine and jurisprudence governing these distinctive signs, explaing in turn the problems faced when applying its registration with the relevant registry office. As for the legal recognition, the international doctrine is completely divided, some authors believe that the scent marks should be recognized and protected as such, others meanwhile resist conceive such idea. Similarly, the legal requirements that restrict the entry of hon visible marks around the world, revolves around three basic requirements: distinctiveness, visibility, and graphical representation. In that order, and in relation to the entry into force of the Free Trade Agreement signed by the Dominican Republic-Central America and the United States of America (DR-CAFTA), Honduras joined the legal compendium registration Scent Trademarks into its law therefore meeting the requirements and legal reasoning of the Directorate General of Intellectual Property (DIGEPIH) and the Superintendency of Industry and Trade of Colombia addresses this problem. In fact, both governmental entities may be protecting their declarations of refusal under the threshold of the basic requirements inherent in any brand, which are evident in the Sieckman judgment despite the explicit recognition of distinctive odors in the inside of their legal briefs signs. Anyway, the research aims to establish a comparison between Colombia and Honduras in relation to those marks, on the law concerning the law, doctrine and international jurisprudence analysis.

Key words: olfactory mark, distinctive signs, directorate general of intellectual property, superintendence of industry and commerce, distinctiveness, graphic representation, and perceptibility. 


\section{INTRODUCCIÓN}

Ante un inminente e indetenible proceso de cambios globales, especialmente en cuanto a derecho marcario se refiere, los signos olfativos constituyen actualmente un novedoso e interesante tema de estudio tanto en el ámbito nacional como internacional.

Grandes emporios vinculados a la industria, la tecnología y el marketing internacional, buscan incansablemente una forma segura de que sus marcas odoríficas puedan ser objeto de protección, evitando así que sus productos, bienes o servicios sean duplicados.

Recientes estudios ${ }^{2}$ demuestran que el ser humano es capaz de recordar el $1 \%$ de lo que toca, el $2 \%$ de lo que oye, el $5 \%$ de lo que ve, el $15 \%$ de lo que degusta y el $35 \%$ de lo que huele, concluyendo así que un olor o fragancia es capaz de generar múltiples efectos evocativos en la conducta humana (Lannini, 2010).

Desde otra perspectiva, una marca en general puede ser percibida únicamente a través del sentido de la vista, sin embargo, los signos olfativos poseen la exclusiva particularidad de ser percibidos a través del sentido del olfato y al igual que todo signo marcario, estos son utilizados para diferenciar productos, bienes y servicios, de entre otros de su misma clase o especie.

En tal sentido, la protección de una marca se adquiere mediante su registro ante las oficinas de propiedad industrial, no obstante, dicho resguardo se torna imposible cuando los requisitos para su inscripción no superan los estándares establecidos por las entidades encargadas de formalizar su inmediato registro. Al respecto, conviene decir que alrededor del mundo son pocas las marcas olfativas inscritas y los argumentos jurídicos que desestiman su registro varían de país a país, aunque el común denominador para negarlo descansa en tres pilares fundamentales: el primero vinculado a su carácter distintivo, el segundo a su perceptibilidad y el tercero a la forma en que estos pueden ser representados (gráficamente).

El 3 de marzo de 2005, mediante Decreto Legislativo No.10-2005, Honduras suscribió el Tratado de Libre Comercio entre la República Dominicana, Centroamérica y los Estados Unidos (DR-CAFTA), el cual entró en vigencia el 1 de abril de 2006. Dicho

2 Reconocidas empresas, como Channel, han buscado registrar sus productos odoríficos, sin obtener éxito alguno. 
convenio preceptúa en uno de sus apartados lo siguiente: "Artículo 15.2. Marcas. 1. Cada parte dispondrá que las marcas incluirán las marcas colectivas, de certificación y sonoras y podrán incluir indicaciones geográficas y marcas olfativas (...)".

Dado que tal disposición les confiere una expresa inclusión a estos signos marcarios, resulta de vital importancia conocer de qué manera la Dirección General de Propiedad Intelectual de Honduras está ventilando la inscripción de marcas olfativas, cuáles son los requerimientos para alcanzar su protección y qué tipo de exámenes 0 análisis tienen que superar dichas marcas ante esta Dirección General de Propiedad Intelectual.

Resulta importante señalar que Honduras actualmente no posee marcas olfativas inscritas ni se han presentado hasta la fecha solicitudes al respecto (DIGEPIH, 2014), pese a la aprobación del mencionado tratado internacional. En este contexto y con relación a la sentencia emitida por el Tribunal de Justicia Europeo en cuanto a marcas olfativas se refiere -el pronunciamiento Sieckman y las resoluciones emitidas por la Superintendencia de Industria y Comercio de Colombia-, la Dirección General de Propiedad Intelectual de Honduras estaría sustentando sus pronunciamientos bajo el umbral de los requisitos de perceptibilidad, distintividad y representación gráfica, siendo la doctrina y la jurisprudencia internacional las herramientas más recurrentes en las cuales estas instituciones amparan sus argumentos denegatorios, pese al expreso reconocimiento de los olores en sus compendios jurídicos.

\section{METODOLOGÍA}

Esta investigación proporciona una actualización de la bibliografía en donde la doctrina, las leyes y la jurisprudencia nacional e internacional se interrelacionan armónicamente, examinando además la aplicabilidad y posibilidad que tienen las marcas olfativas de ser inscritas ante las oficinas registrales correspondientes a través de la metodología del derecho comparado.

Se trata de una investigación descriptiva y documental, en la que se aplicó el método teórico, inductivo y analítico, dado que fue más allá de una escueta lectura de fallos y pronunciamientos emitidos por autoridades administrativas; se buscó analizar los razonamientos convergentes entre la doctrina y la jurisprudencia internacional, en donde subyacen los argumentos más controvertidos con relación al tema. 
No obstante, seleccionar dentro de un universo de antecedentes bibliográficos tan amplio, constituye una ardua labor para un solo investigador, por lo tanto, se procedió a distinguir a los signos olfativos dentro de la gama de marcas no tradicionales, determinando así la ubicación y delimitación del tema escogido, como también la construcción de un metódico estudio comparado entre Honduras y Colombia, quien en los últimos años y desde la aprobación de la Decisión Andina 486 se ha enfrentado ante dicha problemática.

La investigación busca analizar y sintetizar los escritos vinculados al tema de las marcas olfativas desde una perspectiva nacional e internacional, tomando como referente jurídico las normas, doctrina y jurisprudencia que rigen a estos signos distintivos, para dilucidar la problemática que enfrentan al solicitar su respectiva inscripción ante las oficinas registrales correspondientes.

Asimismo, con la entrada en vigencia a Honduras del Tratado de Libre Comercio (DR-CAFTA) se examinará la forma, contenido y procedimientos científicos con que la Dirección General de Propiedad Intelectual y la Superintendencia de Industria y Comercio podrían estar utilizando para verificar la distintividad, perceptibilidad y representación gráfica de estos signos marcarios.

Al respecto conviene decir que ambos países (Honduras y Colombia) podrían estar sustentando sus resoluciones al amparo de los criterios jurídicos emitidos por el Tribunal de Justicia Europeo mediante la sentencia Sieckman.

Sinopsis sobre las marcas olfativas y su protección jurídica

Los argumentos jurídicos en el campo internacional para objetar o conceder la inscripción de una marca odorífica convergen en una doble vía; la primera de carácter interpretativa, porque suele desplegarse en un contexto de legislaciones y doctrinas permisibles; la segunda es de carácter restrictiva, porque devela requerimientos subjetivos que giran en torno a la distintividad, perceptibilidad y su controvertida desaprobación en cuanto a la forma gráfica en que estas deben ser representadas.

\section{Alemania}

Uno de los precedentes jurisprudenciales a escalas internacionales lo constituye la sentencia Sieckman, emitida por el Tribunal de Justicia de la Comunidad Europea (TJCE) el 12 de diciembre de 2002, en la cual un interesado pretendió inscribir un signo olfativo identificado como balsámico afrutado con ligeras reminiscencias a canela, sin lograr su cometido al no satisfacer los criterios exigidos por aquel Tribunal 
(Porras, 2005).

Dicha sentencia refleja diversos matices en cuanto a las argumentaciones jurídicas que versan en torno a los requisitos exigidos por el Tribunal de Justicia Europeo (TJCE), generando un efecto de carácter vinculante y de guía argumentativa no solo para aquellos países miembros de la Comunidad Europea, sino también para la jurisprudencia y legislación internacional que afrontan la misma problemática en cuanto a la protección de marcas olfativas.

\section{Francia}

Cada país de la Unión Europea cuenta con un sistema marcario diferente y es de público conocimiento que Francia constituye uno de los emporios más atractivos en cuanto a la manufactura de perfumes se refiere; por tanto, este país debería ser una de las naciones con mayor número de marcas olfativas registradas, no obstante, la realidad jurídica supera los intereses económicos.

Esta nación, al ser parte de la Unión Europea, provoca que su derecho marcario se vea influenciado por los criterios jurisprudenciales del Tribunal de Justicia Europeo; en esta vía restrictiva han sido publicadas cinco solicitudes de marcas olfativas en el Registro de la Propiedad Industrial de Francia, pero todavía ninguna ha sido inscrita (Ruiz-Jarabo, 2001).

\section{Estados Unidos}

Esta nación es una de las potencias que más adelantos evidencia en cuanto al registro de marcas olfativas, es considerado como el primer Estado que registró ante sus oficinas una marca odorífica. Esta solicitud fue requerida para la inscripción de un hilo de coser y bordar, conocido como el asunto "In re Clarke", descrita así: "a high impact, fresh, floral fragrance reminiscent of plumeria blossoms" (Ruiz-Jarabo, 2001). Asimismo, este país ha logrado incorporar ante los registros correspondientes las marcas olfativas siguientes:

1. US 2560618 «The scent of bubble gum», fragancia registrada para aceites industriales.

2. US 2463044 «The cherry scent», aroma adherido a lubricantes sintéticos.

4. US 2596156 «The strawberry scent», esencia destinada a lubricantes, aceites y combustibles de motor (Balañá, 2006, p. 21).

5. 3140700 (scent only), este perfume con olor a menta es incorporado a productos de oficina, carpetas de archivo y carpetas colgantes (Erick Pelton \& Associates, 2011). 


\section{Argentina}

Este país no restringe la inscripción de marcas odoríficas, puesto que la compañía L'Oréal después de varios intentos logró conseguir el primer registro de aromas (números 2.270.653/54/55/56 a 2.270.657 bajo la Clasificación 3 de Niza), comprendiendo dentro de ellas $A$ diferentes fragancias aplicadas en frascos que evocan diversos aromas frutales (Allman y Zuccherino 2010).

\section{Reino Unido}

En una primera etapa este país admitió el registro de dos marcas olfativas, identificadas bajo la numeración 2001416 y 2000234 . La primera describe el olor de rosas aplicado a neumáticos y la segunda el olor de cerveza para distinguir dardos; últimamente, la United Kingdom Trade Mark Registry ha denegado la inscripción de una marca consistente en el aroma o la esencia de canela para distinguir ciertos mobiliarios y sus complementos (Ruiz-Jarabo, 2001).

\section{España}

Las marcas olfativas en este país no pueden ser objeto de inscripción, basándose en el argumento de que un olor no puede ser representado gráficamente, así lo dispone la Ley de Marcas Española, LME, la cual en su Título II, Capítulo I, Artículo 4, Numeral 1 , define que una marca es todo signo susceptible de representación gráfica que sirva para distinguir en el mercado los productos o servicios de una empresa de las de otras (Ley 17/2001, Artículo 4).

\section{Australia}

Este país adopta criterios más prácticos y menos complejos en cuanto al registro de una marca odorífica, en vista de que el requisito imperante en la representación de un olor es la descripción verbal y escrita, dejando de lado cualquier metodología tendiente a analizar de forma científica una fragancia, debido a que estas suelen escapar al entendimiento humano de mediano intelecto (Chijane, 2007).

Doctrina e investigaciones referentes al tema de marcas olfativas

Las marcas olfativas han sido objeto de múltiples y debatidas controversias, sobre todo en la esfera internacional, pues las premisas argumentativas con respecto a su protección giran en torno a los requisitos de perceptibilidad, distintividad y, especialmente, al requisito de representación gráfica que lo complementa.

La temática es de gran trascendencia, sin embargo, son pocos los autores que han optado por estudiar a profundidad la materia; en consecuencia, a continuación se 
presenta una actualización de la bibliografía sobre el tema, en la cual se exponen diversos escenarios y tendencias jurídicas.

Cann (1989), Shab (1990) y Morrin y Ratneshwar (2000) realizaron algunos estudios, con propósitos específicos, en donde se buscaba medir la inteligencia a través de la percepción olfativa. La investigación consistió en exponer a un variado grupo de participantes a una serie de artículos, imágenes, palabras o nombres de marcas durante un lapso de tiempo, logrando determinar que ciertos olores podían favorecer considerablemente la memorización y que, además, el recuerdo y el reconocimiento de los artículos mejora de forma considerable, sobre todo en su fase de aprendizaje.

Fernández (2001) y Casado (2000) señalan que los signos olfativos no deberían ser objeto de protección y mucho menos ser considerados como tal; sin embargo, Rizzo (2002) y Senna (2003) estiman que las marcas olfativas deben ser aceptadas y, por ende, reconocidas dentro del ámbito marcario, al igual que cualquier marca en general.

Por su parte, Otamendi (2002) es completamente partidario de concederle a las marcas odoríficas la posibilidad de ser protegidas y registradas, pues al hacerlo se estaría combatiendo la piratería y se evitaría de igual modo que terceros se enriquezcan con la invención de otros, así lo deja entrever en las siguientes palabras:

Nada dice la ley respecto del registro marcario de un determinado olor, aroma o fragancia. Quien dote al envase o envoltorio que usa para vender un producto de un determinado aroma, puede, con todo derecho, querer ser el único en distinguir su producto con el aroma respectivo. Para ello, nada mejor que registrarlo como marca. Es probable que haya quienes sostengan que tal privilegio no es admisible en nuestro sistema marcario (Otamendi, 2002, p. 61-62).

Uribe (2002) verifica una sutil referencia con relación a las marcas olfativas, considerando que el registro de olores responde a los avances tecnológicos actuales y por el carácter distintivo que las envuelve, tendrían que difundirse a merced de una denominación ante la inminente dificultad de encontrar una fragancia que sea suficientemente característica, no usual o connatural al producto y no se encuentre dentro del dominio público.

Bertone y Cabanellas (2003) aportan un tratamiento específico sobre marcas odoríficas en el Libro I, en este primer tratado se aborda en un subtítulo denominado "Signos no susceptibles de representación", indicando que frente a la imposibilidad de 
superar esa representación gráfica, no constituye un obstáculo insuperable para que un signo pueda ser calificado como marca. Sin embargo, el procedimiento de registro que la ley prevé y la práctica que de tal procedimiento se hace, obran como impedimento para el registro como marcas de los signos no susceptibles de representación gráfica, sin perjuicio de la posibilidad de que operen válidamente -con los consiguientes efectos jurídicos- como marcas de hecho.

Al respecto, cabe determinar qué signos son calificables como no susceptibles de representación gráfica. Respecto de los signos sonoros, en ciertos casos no presentan dificultades prácticas para su representación gráfica-como sucede con los signos musicales, como los pentagramas-; mientras que para otros -rugidos, chirridos, etc.- su representación puede ser prácticamente imposible. En estos casos, frente a la falta de una prohibición en el derecho argentino, debería ser factible una descripción gráfica o verbal del sonido, acompañada por una grabación del mismo (Otamendi, 2002, p. 59).

"Respecto de las marcas olfativas, táctiles y gustativas, si bien tienen en ciertos casos potencialidad distintiva -limitada por su contenido funcional-, presentan en el sistema actual dificultades sustanciales de representación gráfica y aún de identificación, que las hacen incompatibles con los mecanismos contemporáneos de registro" (Otamendi, 2002, pp. 434-435)

Antequera (2009) detalla un variado compendio de marcas no tradicionales, de las que se pueden mencionar las sonoras, animadas y especialmente las tridimensionales, profundizando en aquellos aspectos contentivos a la distintividad y perceptibilidad exigidas para su inscripción y registro. No se hace ningún planteamiento en esta obra que indique o señale a las marcas olfativas como tema de estudio.

Liévano (2011) efectúa un interesante y profundo estudio respecto a las marcas no tradicionales y los requerimientos que definen a las mismas definiendo, de forma individual y a la vez específica, los aspectos referentes la distintividad, perceptibilidad y representación gráfica. Con relación a la distintividad de las marcas olfativas, dicho autor sostiene que las fragancias en sí mismas no poseen la capacidad innata de hacer distintivo un producto y que basados en la opinión de la Oficina de Armonización del Mercado Interior (OAMI), la representación gráfica utilizada por algunos en ningún momento satisface los requerimientos exigidos por la mayoría de registro de la Unión Europea.

Chijane (2007) enmarca el tema de las marcas olfativas bajo la denominación de las 
nuevas marcas y divide su escrito entre las posiciones a favor y en contra del registro de signos olfativos, la carencia de distintividad, duración del aroma y la imposibilidad de ser representadas gráficamente. Los argumentos que respaldan su inscripción radican en que si los aromas representan a un signo distintivo como palabras 0 grafías, no existe razón alguna para excluirlas dentro de esa categoría. En cuanto a la representación gráfica y su dificultad de cumplir con este requisito procedimental, debería dejarse a un lado tal rigidez y permitir la descripción escrita que se realiza del olor; es más, podrían admitirse técnicas de fraccionamiento mediante las cuales se aíslan los componentes volátiles del olor para luego identificar cada uno de ellos, comparándolos con la base de datos de compuestos conocidos, como la cromatografía de gases y la cromatografía líquida de alto rendimiento. No obstante, dicha técnica podría ser contraproducente debido al nivel de distintividad que un aroma vislumbra y que una persona de mediana inteligencia pudiese distinguir dentro de otras fragancias de su misma clase o especie. En tal sentido, la imposibilidad de que estas marcas puedan ser representadas gráficamente representa el mayor inconveniente para las oficinas de propiedad industrial, puesto que al no cumplirse con tal requerimiento, estas pueden ser objeto de rechazo frente a la alternativa de la descripción verbal, cuyo argumento denegatorio podría sustentarse en que la misma no es clara, precisa u objetiva.

Marcas olfativas desde la perspectiva del derecho comparado: Honduras-Colombia

Con el propósito de proveer un estudio comparativo con relación al tema, se tomó como referente jurídico a Colombia, puesto que además de ser un estado que reconoce el registro de marcas olfativas, ostenta un enriquecedor y amplio compendio de resoluciones en donde se razonan los puntos sensibles tratados en la presente investigación.

\section{Apreciación de la Superintendencia de Industria y Comercio de Colombia (SIC)}

En Colombia, las marcas olfativas se encuentran reguladas expresamente en el Artículo134 de la Decisión Andina 486, inciso c), el cual establece lo siguiente: "A efectos de este régimen constituirá marca cualquier signo que sea apto para distinguir productos o servicios en el mercado. Podrán registrarse como marcas los signos susceptibles de representación gráfica... podrán constituir marcas, entre otros, los siguientes signos... c) los sonidos y los olores...".

Bajo ese contexto, Colombia admite la inscripción de marcas olfativas en su legislación nacional, sin embargo, desde que la Decisión Andina 486 fue aprobada en 
el año 2000, no existe a la fecha una tan sola marca olfativa inscrita; las causas 0 razonamientos que la sustentan se ven reflejadas en las resoluciones emitidas por Superintendencia de Industria y Comercio (SIC), entidad encargada de revisar, analizar y registrar todo lo referente al tema de propiedad intelectual e industrial.

Basándose en lo anterior, cabe preguntarse cuáles son las motivaciones que limitan y han restringido la inscripción de una marca olfativa ante la Superintendencia de Industria y Comercio de Colombia durante los últimos 13 años, tomando como referente jurídico las resoluciones 11- 30326-2-0-2011-05-13 y 00017175, la legislación interna y la doctrina nacional.

Para dilucidar el anterior cuestionamiento, resulta oportuno analizar los tres requisitos fundamentales en que descansan las marcas olfativas y que constituyen, a su vez, los tres grandes obstáculos para estas puedan ser inscritas ante la Superintendencia de Industria y Comercio, es decir, la: perceptibilidad, distintividad y representación gráfica.

De conformidad a los pronunciamientos emitidos por la Superintendencia de industria y Comercio, las marcas olfativas presentadas ante la División de Signos Distintivos, han sido denegadas en razón de los argumentos siguientes:

1. Distintividad: es un requisito inherente a cualquier tipo de marca; sin esta, un signo marcario no tendría razón de ser y, por ende, carecería de capacidad diferenciadora. De acuerdo al Tribunal de Justicia de la Comunidad Andina, la distintividad es aquella que:

...Le permite al consumidor realizar la elección de los bienes que desea adquirir, pues el signo marcario le da a la mercadería su individualidad y posibilita que un producto sea reconocido de entre otros similares que se ofertan en el mercado por sus competidores. (Tribunal de Justicia de la Comunidad Andina, 2005).

Este requisito ha generado incontables posiciones doctrinales ante la imposibilidad de distinguir una fragancia de otra, ya que la descripción verbal y la fórmula química del olor no constituyen, según sus opositores, elementos suficientes para superar los exámenes requeridos.

2. Perceptibilidad: a diferencia de todas las marcas restantes, tradicionales y no tradicionales, los signos olfativos invisten la exclusiva particularidad de ser percibidos a través del olfato. Bajo este referente, estudios recientes demuestran que 
los aromas provocan poderosos efectos en el comportamiento humano (relajar, estimular, asustar, provocar, seducir, etc.) y que, de hecho, la memoria es capaz de distinguir entre más de 10,000 aromas diferentes que influyen en nuestras emociones y sentimientos (lannini, 2010). Por su parte, el Tribunal Andino entiende que la perceptibilidad:

"Hace referencia a todo elemento, signo o indicación que pueda ser captado por los sentidos para que, por medio de estos, la marca penetre en la mente del público, el cual la asimila con facilidad" (Tribunal de Justicia de la Comunidad Andina, 2004).

3. Representación gráfica: este parámetro constituye el trascendental obstáculo que ha esgrimido la Superintendencia de Industria y Comercio, pese a la existencia de visibles contradicciones que existen al respecto. Dicha intendencia señala que:

...La susceptibilidad de representación gráfica consiste en expresiones o descripciones realizadas a través de palabras, gráficos, signos mixtos, colores, figuras, etc., de tal manera que sus componentes puedan ser apreciados en el mercado de productos (Superintendencia de Industria y Comercio, 2011).

Partiendo de la premisa que representar gráficamente consiste en expresar o describir mediante palabras, gráficos, signos mixtos, colores y figuras, resulta inexplicable como un gráfico en donde se puntualiza la composición química de un olor, acompañada de una descripción verbal-escrita, no puede ser considerada como tal; algunas legislaciones han aceptado una delineación escrita (caso Australia), por eso se defiende la idea de que pueda aceptarse la composición química como forma de representar gráficamente un aroma.

Consecuentemente, no existe en Colombia una sola marca olfativa inscrita que haya superado la terna de requerimientos exigidos, deduciendo que o no se pueden registrar 0 a pesar de esta posibilidad la oficina encargada ha edificado una fuerte barricada para no permitirlo.

Apreciación de la Dirección General de Propiedad Intelectual de Honduras (DIGEPIH)

En nuestro país, la Ley de Propiedad Industrial no contempla la inscripción de marcas olfativas, así lo expresa el Artículo 79, inciso 2), al conceptualizar a las marcas en la forma siguiente: "Artículo 79. Para los efectos de la presente Ley se entenderá por: ...2) Marca, cualquier signo visible apto para distinguir los productos o los servicios de una empresa, con respecto a los productos o servicios de otras empresas...". 
Evidentemente, la Ley de Propiedad Industrial excluye a las marcas no visibles (sonoras, olfativas, gustativas) dentro del compendio de marcas propiamente dichas a lo interno de su legislación, quedando estos signos distintivos exentos de protección alguna. Sin embargo, al incorporase en Honduras el Tratado de Libre Comercio suscrito entre la República Dominicana, Centroamérica y los Estados Unidos (DR-CAFTA), este obliga a nuestro país a extender una brecha significativa con relación a la admisibilidad de marcas olfativas al compendio jurídico hondureño.

Ante esa disyuntiva jurídica, la regla general indica que en caso de existir conflicto de leyes entre una norma interna y un tratado o convención internacional, se aplicará esta última, así lo dispone la Constitución de la República de Honduras al pronunciar lo siguiente: "Artículo 18. En caso de conflicto entre el tratado o convención y la ley, prevalecerá el primero".

Con relación a los alcances y obligaciones que el DR- CAFTA implica, el Artículo 1.4 preceptúa que: "Las partes garantizarán la adopción de todas las medidas necesarias para hacer efectivas las disposiciones de este tratado, incluida su observancia por parte de los gobiernos estatales, salvo que este Tratado disponga otra cosa".

En conclusión y de conformidad a lo expuesto por la Dirección General de Propiedad Intelectual de Honduras, en la actualidad no existen marcas olfativas inscritas ni se han presentado a la fecha solicitudes al respecto (DIGEPIH, 2014), no obstante, dicha Dirección se encuentra esperando la aprobación del reglamento correspondiente donde se contempla el procedimiento y exámenes a verificar al momento en que una marca olfativa u otro signo no visible contemplado en el Artículo 15.2 del DR-CAFTA solicite su inmediato registro.

Sentencia Ralf Sieckman y su efecto vinculante con relación a las marcas olfativas

Uno de los precedentes jurisprudenciales más influyentes a escalas internacionales lo constituye la sentencia Sieckman, la cual fue emitida por el Tribunal de Justicia de la Unión Europea (TJCE), con fecha 12 de diciembre de 2002, en donde un interesado pretendió inscribir un signo olfativo denominado "balsámico afrutado con ligeras reminiscencias a canela", sin lograr su cometido al no satisfacer los criterios solicitados por aquel Tribunal (Tribunal de Justicia de las Comunidades Europeas, 2002).

El fallo pronunciado en la sentencia giraba en torno a tres aspectos básicos: el 
primero de ellos argumentaba que la marca odorífica carecía de capacidad distintiva, tanto intrínseca como extrínseca; en tanto el segundo ponía en manifiesto que dicho olor era imperceptible a la vista humana y que, finalmente, carecía de representación gráfica.

Este último constituye el argumento más sensible de la sentencia, puesto que al momento de solicitar el Sr. Sieckman la inscripción de la marca olfativa en mención, adjuntó a la misma una descripción escrita, el depósito de una muestra del aroma, como también un gráfico que detallaba su composición o fórmula química. Una vez examinadas, el TJCE procedió a desvirtuar los elementos acompañados, ya que según este la descripción de la fragancia no era clara, precisa u objetiva, la muestra o depósito no era suficiente ni duradero y, por ende, la representación gráfica se limitaba a describir su composición o fórmula química y no a la fragancia en sí (Tribunal de Justicia de las Comunidades Europeas, 2002).

Se observa que los mismos argumentos utilizados por el Tribunal de Justicia alemán son reproducidos en Colombia, a través de la SIC, para denegar la inscripción de una marca olfativa, sobre todo por el requisito de representación gráfica, la cual no puede ser descrita mediante una fórmula química que no representa fehacientemente un aroma y que no podría ser depositada en un frasco como una muestra, puesto que al transcurrir el tiempo este perdería su efecto aromatizante.

Desde el punto de vista institucional, la Superintendencia de Industria y Comercio (SIC), como entidad encargada de registrar marcas en Colombia, se ha confrontado con iguales objeciones con respecto a la inscripción de esta clase de signos distintivos, prueba de ello es que dicha institución ha denegado la inscripción de algunos olores bajo la premisa de que los mismas no superan los estándares de perceptibilidad, distintividad y representación gráfica, (radicaciones 11-30326-2-0-2011-05-13, 00017175 y expediente 05-47635) invocados y fundamentados en la sentencia Sieckman, lo que viene a confirmar que ese precedente jurisprudencial ha traspasado fronteras y, por ende, influenciado de alguna u otra forma a la autoridad competente.

Visto de esa forma y aunque en Honduras no existen marcas olfativas inscritas o se hayan presentado solicitudes ante la Dirección General de Propiedad Intelectual, la norma, doctrina y la jurisprudencia internacional, apuntan fehacientemente a que dicha institución podría amparar en un futuro no muy lejano sus criterios jurídicos a la luz de los mismos argumentos descritos en la sentencia Sieckman y las consideraciones legales de la Superintendencia de Industria y Comercio de Colombia. 


\section{CONCLUSIONES}

1. Las marcas odoríficas constituyen un reto innovador para el derecho de propiedad industrial en general, puesto que al no ser percibidas mediante el sentido de la vista, estas no logran obtener en la mayoría de países su inscripción y registro.

2. Pese a que la decisión Andina 486 de Colombia reconoce expresamente la inscripción de los olores como signos marcarios, la Superintendencia de Industria y Comercio ha señalado que a pesar de ello, los olores no son susceptibles de representación gráfica. Mientras tanto, algunos acuerdos internacionales giran en torno a reconocer o prohibir los olores como signos marcarios, lo cual representa una clara disyuntiva registral.

3. La Superintendencia de Industria y Comercio no hace más que reproducir las restricciones adoptadas por el resto de países alrededor del mundo tendientes a limitar la inscripción de marcas olfativas. Pese a que el Artículo 134 de la Decisión Andina 486 es novedoso en teoría, la práctica jurídica refleja todo lo contrario, ya que requisitos como la representación gráfica y aún más la interpretación que de ella se realiza, frenan el expedito registro de los olores.

4. La doctrina internacional denota una marcada y dual tendencia en cuanto a la inclusión y protección de los signos olfativos en el derecho marcario; por una parte, algunos autores defienden la idea de incorporar esta clase signos en el sistema marcario, mientras que en contradicción, otros se oponen radicalmente a la inclusión de los olores ante la imposibilidad de ser representados gráficamente.

5. Desde que el Tratado de Libre Comercio DR-CAFTA entró en vigencia en Honduras y admitió la inscripción de marcas olfativas, al día de hoy no existen signos odoríficos inscritos por diversas razones; una de ellas es porque ningún solicitante se ha hecho presente ante las oficinas de la Dirección General de Propiedad Intelectual a solicitar su inscripción y, segundo, porque dicha institución no posee a la fecha un reglamento establecido y aprobado en donde se consagren aquellos requerimientos indispensables que permitan su expedito registro.

6. Pese a no existir en Honduras marcas olfativas registradas y la carencia de un reglamento que permita examinarlas, no es de extrañarse que los argumentos jurídicos en que la Dirección General de Propiedad Intelectual de Honduras 
(DIGEPIH) pueda en un futuro próximo estar sustentando sus resoluciones al amparo de los indicadores que la jurisprudencia y doctrina internacional establezcan.

7. Finalmente, la sentencia Sieckman refleja diversos matices en cuanto a sus argumentaciones jurídicas que versan en torno a los requisitos exigidos por el TJCE, generando un efecto de carácter vinculante no solo para aquellos países miembros de la Comunidad Europea, sino que también para la jurisprudencia y legislación internacional que afrontan la misma problemática en cuanto a la protección de estos signos olfativos.

\section{REFERENCIAS}

Antequera Parilli, Ricardo. (2009). Estudios de derecho industrial y derechos de autor. Bogotá: Temis.

Balañá, Sergio. (2006). El entorno digital, ¿segunda oportunidad para la marca olfativa? Estudio acerca de la capacidad del signo olfativo para funcionar como marca en el mercado. España: Universidad de Santiago de Compostela, Instituto de Derecho Industrial. Recuperado de: http://www.marcialpons.es/static/pdf/ 100785277.pdf, pp. 21.

Bertone, Luis Eduardo y Cabanellas de las Cuevas, Guillermo. (2003). Derecho de marcas. Marcas, designaciones y nombres comerciales. Buenos Aires: Editorial Heliasta.

Cann, A. (1989). Olfactory stimuli as context cues in human memory. American Journal of Psichology, (1)102, 91-102. Recuperado de: http://www.ncbi.nlm.nih.gov/pubmed/2929788

Casado Cerviño, Alberto. (2000). El sistema comunitario de marcas: normas, jurisprudencia y práctica. España: Lex Nova.

Chijane, Diego. (2007). Derecho de marcas. Función y concepto-nulidades registro, representación gráfica. España: Editoriales Reus y $\mathrm{B}$ y $\mathrm{F}$.

Fernández, Novoa. (2001). Tratado sobre derecho de marcas. España: Editorial Marcial Pons.

Iannini, Maxi. (2010). Marketing olfativo, un valor diferencial. MK Marketing+Ventas, 253, 60. Recuperado de: http: ubib8.florida-uni.es/textocompleto/marketing... /MK201025308.pdf

Liévano, José Daniel (2011). Aproximación a las marcas no tradicionales. Revista de Derecho Privado, 45. Bogotá. Recuperado de: http://derechoprivado.unian- 
des.edu.co/components/com_revista/archivos/derechoprivado/ pri56.pdf Morrin, M. \& Ratneshwar, S. (2000). The impact of ambient scent on evaluation, attention and memory for familiar and unfamiliar brands. Journal of business recerca, 157-165. Recuperado de: http://econpapers.repec.org/article/eeejbrese Iv_3a49_3ay_3a200 0_3ai_3a2_3ap_3a157-165.htm

Otamendi, Jorge. (2002). Derecho de marcas. Argentina: Lexis Nexis.

Porras, Álvaro. (2005). Marcas olfativas, ¿por qué no? España: Universidad de Alicante. Recuperado de: www.uaipit.com/files/ publicaciones/0000002047_olores.pdf

Rizzo, Sergio y Sandri, Stefano. (2002). Non Conventional Trade Marks and Community Law. Marques. Recuperado de: http://www.wipo.int/cgi-bin/koha/opac-detail.pl?bib=14272

Ruiz-Jarabo, Colomer. (2001). Asunto C-273/00 Ralf Sieckmann contra Deutsches Patent- und Markenamt. Recuperado de:http:// curia.europa.eu/juris/document

Schab, F. (1990). Odor and the remembrances of things past. Journal of experimental Psichology: Learning Memory and Cognition, 16, 648-655. Recuperado de: http://psycnet.apa.org/index.cfm?fa= buy.optionToBuy\&id=1990-27511-001

Senna, Gustavo. (2003). Confusión por asociación. Conferencia del Congreso ASIPI. México.

Tribunal de Justicia de la Comunidad Andina. (2004). Proceso No. 132-ip-2004. Marca: Diacom. Recuperado de: http://www.tribunalandino.org.ec/sitetjca/index.php?option=com_wrapper\&view= wrapper\&ltemid=23

Tribunal de Justicia de la Comunidad Andina. (2005). Proceso No. 94-ip-2005. Marca:

Epomega. Recuperado de: http://www.tribunalandino.org.ec/sitetjca/dex.php? option=com_wrapper\&view= wrapper\&ltemid=23

Tribunal de Justicia de las Comunidades Europeas. (2002). Caso Ralf Sieckman c. Deutsches Patent - und Markenamt. Asunto C-273/00. Recuperado de: http: oami.europa.eu/es/mark/aspects/ pdf/ JJ000273.pdf

Uribe Arbeláez, Martín. (2002). Propiedad Industrial, neoliberalismo y patente de la vida. Bogotá: Ediciones Doctrina y Ley. 